Check for updates

The BMJ

Cite this as: $B M J 2022 ; 376: 0373$ http://dx.doi.org/10.1136/bmi.0373 Published: 14 February 2022

\title{
Type 1 diabetes drug was withdrawn because of a "commercial conflict of interest," charity argues
}

\section{Elisabeth Mahase}

Pharmaceutical company AstraZeneca has withdrawn the type 1 diabetes indication for its drug dapagliflozin because of fears that required changes to the product label would cause confusion among doctors when prescribing it for other conditions.

The company voluntarily removed the type 1 diabetes indication for $5 \mathrm{mg}$ dapagliflozin in October 2021 after $\mathrm{UK}$ and EU medicines regulators advised that-despite there being no new safety or efficacy concerns-an inverted black triangle would need to be added to the label to signify that additional monitoring is required when this drug is prescribed.

Despite the use of the black triangle not being uncommon, AstraZeneca said in its withdrawal announcement that the changes "might cause confusion among physicians treating patients with type 2 diabetes, heart failure with reduced ejection fraction, or chronic kidney disease."

But the Juvenile Diabetes Research Foundation (JDRF), a non-profit organisation that funds type 1 diabetes research, said AstraZeneca's actions were driven by a "commercial conflict of interest," as other larger patient groups had been prioritised at the expense of those with type 1 diabetes.

Speaking to The BMJ, JDRF policy director Hilary Nathan said, "Dapagliflozin is an important treatment for people with type 1 diabetes in helping to reduce blood glucose levels and prevent the heightened risk of longer term cardiovascular and renal complications. We believe that there is a commercial conflict of interest that is driving such decision making. In particular, we are concerned that the decision to withdraw this treatment lacks clinical scrutiny and regulatory oversight to establish the impact on health outcomes for people with type 1 diabetes."

JDRF has also warned that the move could have wider implications, as it sets a precedent by which a commercially driven drug company can remove access to a drug without any scrutiny of potential conflict of interests or cross-sector consultation.

Diabetes UK director of health intelligence and professional liaison, Simon O’Neill, said, “We're disappointed that AstraZeneca and the Medicines and Healthcare Products Regulatory Agency could not find a solution that allowed people living with type 1 diabetes to continue to use the drug safely. If it was possible to find a way of doing so, without causing potential confusion to people with diabetes or healthcare professionals, then we would like to see dapagliflozin reinstated.”

\section{Black triangle awareness}

While the use of the inverted black triangle is a relatively common practice with drugs new to the European market or those that have a new indication added, ${ }^{1}$ research by the European Medicines Agency found that only $51 \%$ of the 2918 people asked said they had seen the black triangle and the accompanying statement before.

Awareness of the symbol also varied between different respondents, with the lowest awareness among patients (30\%) and the highest among pharmacists (83\%). Additionally, only $36 \%$ (1050 of 2918 responders) of responses showed an acceptable understanding, while $17 \%$ of the responders were assessed as misunderstanding. Among those who misunderstood, the most frequently mentioned themes were safety concerns or lack of safety data. ${ }^{2}$

While acknowledging potential awareness problems around the black triangle, diabetes and endocrinology consultant Sufyan Hussain-a member of JDRF UK's scientific advisory council-said the way the withdrawal was handled shows that "better stakeholder engagement with those living with the condition by industry is needed."

He told The BMJ, "Efforts to understand those affected and preserve the option in type 1 diabetes or those with hybrid type 1 and type 2 diabetes phenotypes, such as considering different branding with a licence in type 1 diabetes, could have been explored. While there is a cost, it may have allowed a positive step for all involved."

Hussain added that he would like to see more transparency and better communication around the decisions pharmaceutical companies are making, as while he understands that companies have investors to satisfy, it's important good research and intellectual property are not lost because of commercial decisions.

When contacted by The BMJ, AstraZeneca did not respond to the accusation that it had prioritised the larger and more profitable patient groups over patients with type 1 diabetes, but referred the journal to the statement the company made at the time of the indication withdrawal (see box for full statement).

MHRA chief safety officer, Alison Cave, said, “The indication for dapagliflozin $5 \mathrm{mg}$ in the treatment of patients with type 1 diabetes mellitus was voluntarily withdrawn by AstraZeneca in the UK and across Europe. This was not linked to any new safety concerns.

"We sought advice on the withdrawal of the indication from independent UK experts. Before we made our own communications, we talked to diabetes 
charities to understand their concerns and how best we can reassure patients. We will continue to engage with stakeholders to understand the impact on UK patients. We have taken steps to reassure patients that the other uses of dapagliflozin are unchanged.”

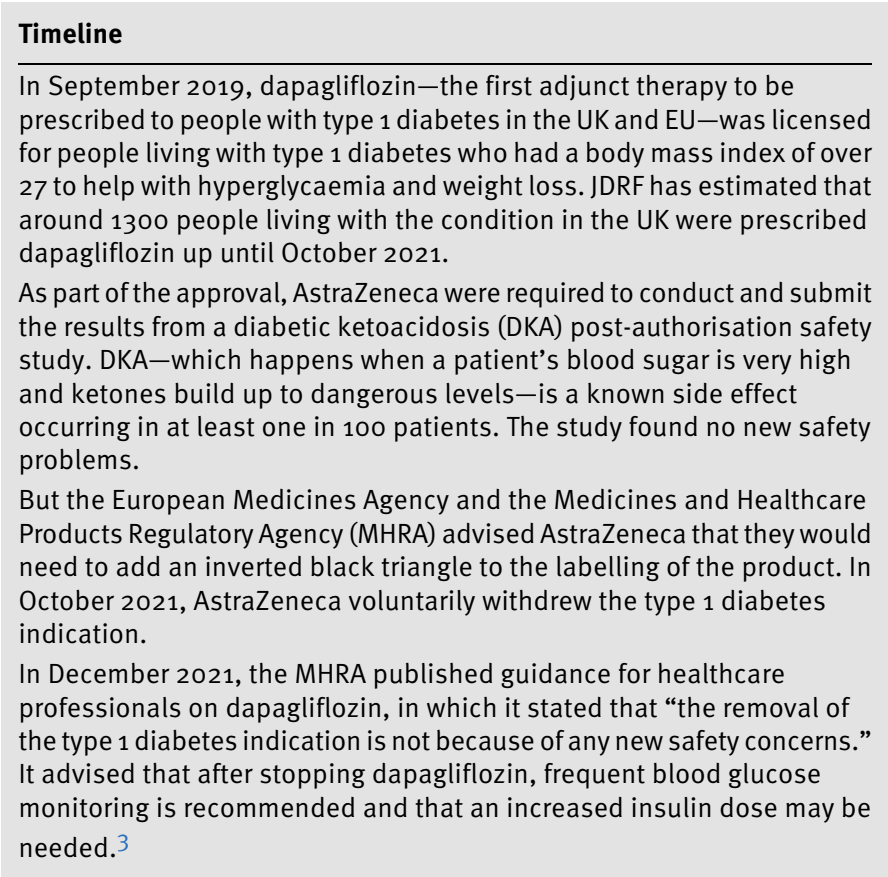

\section{AstraZeneca's statement in full}

AstraZeneca has taken the decision to voluntarily remove the indication for Forxiga $5 \mathrm{mg}$ (dapagliflozin) in the UK, for the treatment of adults with insufficiently controlled type 1 diabetes $\left(\mathrm{T}_{1} \mathrm{D}\right)$. This decision was not due to any new safety or efficacy concerns in $\mathrm{T} 1 \mathrm{D}$ or any other indication. It also does not impact other approved dapagliflozin indications or the 10 $\mathrm{mg}$ dose within or outside of the UK.

This decision has been agreed with the Medicines and Healthcare Products Regulatory Agency for Great Britain and the European Medicines Agency for Northern Ireland. It follows discussions regarding product information changes needed post-approval for dapagliflozin $5 \mathrm{mg}$ specific to $\mathrm{T} 1 \mathrm{D}$, which might cause confusion among physicians treating patients with type 2 diabetes, heart failure with reduced ejection fraction, or chronic kidney disease.

Patients with $\mathrm{T} 1 \mathrm{D}$ currently being treated with dapagliflozin should consult with their healthcare professional regarding their ongoing care.

1 Medicines and Healthcare Products Regulatory Agency. The black triangle scheme. 11 December 2014. www.gov.uk/drug-safety-update/the-black-triangle-scheme-or.

2 European Medicines Agency. European Medicines Agency and member states joint report to the European Commission on the experience with the list of products subject to additional monitoring. 8 March 2018. www.ema.europa.eu/en/documents/report/european-medicines-agency-memberstates-joint-report-european-commission-experience-list-products_en.pdf.

3 Medicines and Healthcare Products Regulatory Agency. Dapagliflozin (Forxiga): no longer authorised for treatment of type 1 diabetes mellitus. 10 December 2021. www.gov.uk/drugsafety-update/dapagliflozin-forxiga-no-longer-authorised-for-treatment-of-type-1-diabetes-mellitus. 